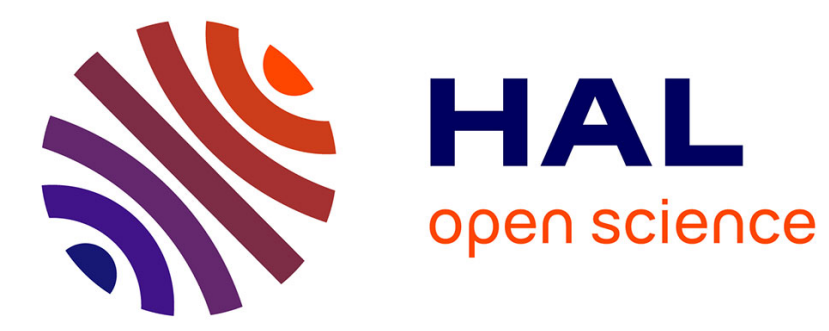

\title{
Eddy current evaluation of air-gaps in aeronautical multilayered assemblies using a multi-frequency behavioral model
}

\author{
Thanh Long Cung, Pierre-Yves Joubert, Eric Vourc'H
}

\section{- To cite this version:}

Thanh Long Cung, Pierre-Yves Joubert, Eric Vourc'H. Eddy current evaluation of air-gaps in aeronautical multilayered assemblies using a multi-frequency behavioral model. Measurement Journal of the International Measurement Confederation (IMEKO), 2011, 44 (6), pp.1108-1116. 10.1016/j.measurement.2011.03.012 . hal-00832676

\section{HAL Id: hal-00832676 https://hal.science/hal-00832676}

Submitted on 11 Jun 2013

HAL is a multi-disciplinary open access archive for the deposit and dissemination of scientific research documents, whether they are published or not. The documents may come from teaching and research institutions in France or abroad, or from public or private research centers.
L'archive ouverte pluridisciplinaire HAL, est destinée au dépôt et à la diffusion de documents scientifiques de niveau recherche, publiés ou non, émanant des établissements d'enseignement et de recherche français ou étrangers, des laboratoires publics ou privés. 


\title{
Eddy current evaluation of air-gaps in aeronautical multilayered
}

\author{
assemblies using multi-frequency a behavioral model
}

Cung Thanh Long - Pierre Yves Joubert - Eric Vourch

\author{
SATIE, ENS Cachan, CNRS, UniverSud, \\ 61, avenue du Président Wilson \\ F-94 235 Cachan Cedex \\ \{thanh-long.cung, pierre-yves.joubert, eric.vourch \}@satie.ens-cachan.fr
}

\section{ABSTRACT}

This paper reports on the estimation of the air-gap thickness between parts in an aeronautical multilayered metallic assembly, by the means of the eddy current method. A behavioural multi-frequency modelling of the interactions between the used cup core sensor and the multilayered structure was developed thanks to the analysis of experimental data, completed with finite element electromagnetic computations. The elaborated model was used to estimate the air-gap thickness (in the range of 0 to $500 \mu \mathrm{m}$ ) of a multilayered structure featured by a metallic coating of known thickness $(1.5 \mathrm{~mm})$ and a distant metallic layer of unknown thickness (in the range $1.5 \mathrm{~mm}$ to $3.5 \mathrm{~mm}$ ). The obtained estimation error is smaller than a few percents, either for simulated or experimental data.

KEYWORDS: Non-destructive evaluation, eddy currents, multi-frequency approach, air-gap estimation, multilayered aeronautical assemblies, behavioural model

\section{Introduction}


The non-destructive evaluation (NDE) of the integrity of conductive parts is a major issue in the manufacturing and in the maintenance of aircrafts, whether for evaluating geometric parameters of pieces during their manufacturing, or for characterizing the defects likely to appear inside in service structures. The eddy currents (EC) technique is well suited to such applications since it is easy to implement, sensitive, robust and eco-aware. The principle of this technique consists in inducing EC inside the inspected structure, for example by means of a coil and, on the other hand, in sensing the response of the structure to the EC excitation, for example through the resulting impedance variations at the ends of the inducting coil, as they reflect the local (either electrical or geometrical) characteristics of the target. Under certain conditions, it allows defects [1,2,3] or geometrical/physical features [4] of the inspected piece to be evaluated with satisfactory accuracy.

However, as far as multilayered structures such as aeronautical assemblies are concerned, the NDE is an "ill-posed" problem the resolution of which is reputed difficult [5], among other reasons because of the incompleteness of the available EC data [6].

To solve this problem, several methods have been proposed. First, analytical models of air-coil sensors coupled with layered structures have been developed $[7,8]$, and used in an inversion scheme to carry out the estimation of the layered structure [9]. Other solutions have been developed using neural networks [10]. Another possibility consists in an experimental approach: the experimental data being directly differentiated from measurements simultaneously carried out on a series of known calibrated mock-ups [11].

In this paper, we propose to build a multi-frequency behavioural model by analyzing the interactions between a cup-core EC sensor and a multilayered assembly. This technique has the advantage of being applicable to any type of EC sensor and to be easy to implement, once the model developed. Moreover, the multi-frequency approach allows the available EC data to be enriched so as to increase the reliability and the accuracy of the NDE [1]. 
We consider the problem of evaluating the thickness of an air layer comprised between two conductive plates like for the NDE of aircraft wings, where a coating, spars and ribs are assembled. Section 2 both reports on the experimental setup and on the analysis of the interactions between a sensor and a layered structure. In Section 3, the experimental analysis is completed by simulations based on finite elements modelling. In Section 4, we propose a behavioural model for the considered problem as well as multi-frequency evaluation algorithms drawn on the analyses presented in the previous sections. Section 5 provides evaluation results obtained under different assumptions. Both the cases of a known and an unknown rib thickness are thus considered, whether using simulated or measured sets of test data. Finally, conclusions are given in section 6 as well as some perspectives to our work.

\section{Experimental study of the problem}

\subsection{Experimental setup}

Typically, the NDE problem that consists in estimating the air-gap between two conductive plates is encountered in aeronautical assemblies such as coatings fixed on wing ribs or spars (Figure 1). In order to experimentally study this problem, an aluminium alloy featuring an electrical conductivity $\sigma=17 \mathrm{MS} / \mathrm{m}$ and a unitary relative magnetic permeability is used.

In the experimental setup, the wing coating is represented by a plate of thickness $t_{c}=1.5 \mathrm{~mm}$, whereas the rib which in practice is a piece of variable thickness (Figure 1), is represented by a series of plates featuring thicknesses comprised between $1.5 \mathrm{~mm}$ and $25 \mathrm{~mm}$. To represent the variable air-gap separating the conductive layers, we use isolating mock-ups featuring both unitary relative magnetic permeability and dielectric permittivity. This gap, denoted $t$, is comprised in the $[0 \mu \mathrm{m} 500 \mu \mathrm{m}]$ range. The EC NDE is performed by means of a 
magnetic cup-core coil placed on the coating, as shown in Figure 2. The coil features 110 turns and an outer diameter of $35 \mathrm{~mm}$. Its free impedance (obtained when the sensor is not coupled with the target) is equivalent to a resistance $R_{0}=4.6 \Omega$ in series with an inductance $L_{0}=3.04 \mathrm{mH}$. The NDE relies on the measurement of the sensor impedance via the lock-in amplification performed by an impedance analyzer (HP4192A) controlled by a computer. More precisely, the particular impedance that we consider is the normalized impedance denoted $Z_{n}$ and defined in [4] for a harmonic as follows:

$$
Z_{n}=R_{n}+j \cdot X_{n}=\left(Z_{t}-R_{0}\right) / X_{0}
$$

where $Z_{t}$ is the impedance of the sensor when placed on a two plates assembly featuring a given air-gap $t$, and $X_{0}$ is the free reactance of the sensor.

\subsection{Sensor and target interactions}

In the case of an air coil placed on a bulk conducting piece, an analogy can be made between the sensor behaviour and a loaded transformer. As shown in [12], the normalized impedance $Z_{n}$ of the sensor is a function of the electromagnetic parameters $(\sigma, \mu)$ of the bulk conductive piece, of its thickness $t_{b}$ and of the EC frequency $f$ :

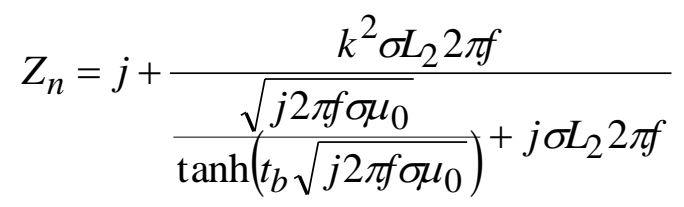


where $k$ is the electromagnetic coupling coefficient between the sensor and the target (which depends on the sensor-target distance), where $L_{2}$ is the self-inductance of the target, which reflects the relationship between the EC induced in the target and the electromagnetic energy of the stored induction. The latter self-inductance is assumed to be constant and independent of both the frequency and the properties of the target [13].

The evolution of $Z_{n}(2)$ in the $\left(R_{n}, X_{n}\right)$ plane, called the universal impedance diagram (UID), have been studied $[4,12]$ in the case of a bulk target.

In our study, the target is a multilayered assembly similar to that of a coating on a wing rib or spar, possibly separated by an air-gap of thickness $t$. Therefore, relation (2) only applies in the case $t=0$. However, it has been shown in previous works [14] that the normalized impedance of the sensor coupled with the multilayered assembly, denoted $Z_{n t}$, is a function of the coating and rib thicknesses $t_{c}, t_{r}$, and of the gap $t$, and is advantageously studied in the UID plane.

The experimental setup is implemented so as to measure the normalized impedance of the sensor over a large frequency range (from $80 \mathrm{~Hz}$ to $30 \mathrm{kHz}$ ) in order to represent the UID associated with $Z_{n t}$. The IUD obtained for an assembly featured by $t=0 \mu \mathrm{m}$ (close to a bulk target) and $t=500 \mu \mathrm{m}$, are shown in Figure 3.

These IUD show that there exists a frequency range in which the properties of the measured EC data are likely to allow $t$ to be evaluated. As shown in Figure 4, which represents the UID obtained in this frequency range with assemblies featuring a gap comprised between 0 and $500 \mu \mathrm{m}$, the distance between the two plates is proportional to that between the two corresponding UID curves. Indeed, the locus of the points obtained at the same frequency but for different gaps $t$ ( $t_{c}$ and $t_{r}$ being fixed) is a linear curve. This result led 
us to analyze the relationship between $t$ and a normalized impedance distance (NID) defined as follows:

$$
N I D=\left|Z_{n t}(f)-Z_{n 0}(f)\right|
$$

where $Z_{n 0}$ denotes the normalized impedance of the sensor obtained when the coating and the rib are in contact (i.e. $t=0$ ), and where $Z_{n t}$ denotes the normalized impedance obtained when the gap is $t$ (assumed nonzero and for the sake of clarity indexed in $\mu \mathrm{m}$ in Figure 4).

It appears experimentally that a linear equation relates NID to $t$ (4), provided that the frequency $f$ does not exceed a maximum value $f_{\max }, f_{\max }$ being such that the skin depth $\delta$ of the induced EC approximately equals $2 / 3$ of $t_{c}$.

$$
N I D(t)=a\left(f ; t_{c} ; t_{r}\right) \cdot t
$$

Further analysis also shows that an optimal frequency $f_{\text {opt }}$ (equal to $1060 \mathrm{~Hz}$ in the studied example) exists, that maximizes the slopes of the $N I D(t)$ linear relations, whatever the value of $t$ (Figure 5). $f_{\text {opt }}$ is such that the skin depth $\delta$ of the EC approximately equals 2.5 times $t_{c}$. As shown in Figure 4, both the modulus and the phase of the normalized impedance are modified by the presence of the air-gap. However, the variations of the phase with the air-gap are small compared to the variations of the modulus. Furthermore, taking into account the sole modulus of the normalized impedance allows a linear model of the sensor / layered structure to be derived. This is why only the modulus of the impedance variation is considered in this study. 
Based on the linear characteristics (4) it is possible to elaborate a gap evaluation method. Prior to this, it is worth noting that in the $\left[f_{\text {opt }} f_{\max }\right]$ range the normalized impedance distance decreases as the excitation frequency increases (Figure 5). To illustrate this point Figure 6 shows the linear curves (4) obtained experimentally at the two frequencies $f=680 \mathrm{~Hz}$ and $f^{\prime}=6600 \mathrm{~Hz}$. Still experimentally, one can also notice that for a fixed coating thickness and a fixed excitation frequency, the thicker the rib the higher the NID.

If the sensor is placed on an aircraft wing at a particular position where both $t_{c}$ and $t_{r}$ are known, it is possible to estimate $t$ from a single $N I D_{i}$ measurement at a frequency $f_{i}$. This requires the knowledge (for example from experimental learning) of the slope $a_{i}$ of the linear characteristic $N I D_{i}(t)$ at $f_{i}$ and for the considered fixed values $t_{c}$ and $t_{r}$. It is thus possible to estimate the air-gap $\hat{t}_{i}$ according to (5).

$$
\hat{t}_{i}=a_{i}^{-1} \cdot N I D_{i}
$$

However, since in the actual considered aeronautical problem the thickness $t_{r}$ is a priori unknown, the knowledge of only one linear characteristic (4) determined at one given frequency is not sufficient to estimate $t$. To solve this problem, a multi-frequency approach is necessary.

\section{Finite elements modelling of the problem}

The use of linear functions (4) can be envisaged for determining $t$. So for, a behavioural model of the interactions between the sensor and the layered assemblies is necessary. 
Therefore, we build a comprehensive database by simulating these interactions in the whole variety of cases considered, using finite elements (FE) modelling implemented by means of the commercial software ANSYS.

Sets of linear plots $N I D(t)$ are thus built considering a fixed coating thickness $t_{c}=1.5 \mathrm{~mm}$ and the five following rib thicknesses $t_{r}$ given in $\mathrm{mm}\{1.5,2.0,2.5,3.0,3.5\}$. For each value of $t_{r}$ we consider that the air-gap separating the coating from the rib can vary from $100 \mu \mathrm{m}$ to $500 \mu \mathrm{m}$ by steps of $100 \mu \mathrm{m}$. Moreover, the simulations are performed for the five following excitation frequencies $f\{680,1060,1440,1820,2200\}$ expressed in Hz. Thus, a set of 125 configurations is covered. Given the axial symmetry of the problem, 2D simulations can be performed in the $(r, z)$ plane, as shown in Figure 7.

In order to reduce computational noise, the three layers structure (coating/air/rib) is divided into elementary sub-layers featuring the same mesh. In this way, and as illustrated in Figure 7, from one simulation to another, it is only the material characteristics (aluminium or air) assigned to the different sub-layers that may be changed according to the considered airgap and rib thickness.

The impedance of the sensor is determined as the ratio between the induced electromotive force emf at the ends of the sensing coil and of the excitation current $i_{\text {exc }}$, as follows:

$$
Z=\frac{e m f}{i_{\text {exc }}}=\frac{j 2 \pi f \phi}{i_{\text {exc }}}
$$

where $\phi$ denotes the magnetic flux sensed by the whole set of turns of the coil:

$$
\phi=\sum_{p} \sum_{q} \phi_{p, q}
$$


where $\phi_{p, q}$ is the magnetic flux sensed by the turn of radius $r_{q}$ located at the height $z_{p}$.

As shown in Figure 8, which compares experimental results to the corresponding simulations, the simulations are in good agreement with the measurements and, as expected, the simulated NID vary linearly as a function of $t$. In these computations, the possible lift-off of the sensor is not taken into account in the simulated configurations. Indeed, in practice, since the sensor is placed in contact with the layered structure, the variations of the distance between the sensor and the layered structure can be assumed to be very small compared to the sensors dimensions, and hence, the lift-off effect can be neglected [15]. However, in order to simulate some variability in the sensor positioning, an additive Gaussian noise of adjustable intensity may be added to the computation results [16].

\section{Air-gap evaluation}

In this section, we consider different categories of the air-gap evaluation problem. First (case A) the sensor is assumed to be placed on the aircraft wing at a particular position where the coating and the rib thicknesses are known. As explained in section 2.2, in such a case, provided the slope $a_{i}$ of a linear characteristic (4) (for example previously determined by experimental learning) at a given excitation frequency $f_{i}$ and for the given values of $t_{c}$ and $t_{r}$, one measurement $N I D_{i}$ is sufficient to estimate of the air-gap according to (5).

Nevertheless, in the actual aeronautical problem $t_{r}$ is a priori unknown and the knowledge of only one linear characteristic (4) determined at one given frequency is not sufficient to estimate $t$. To solve this problem, which we will divide into the two following categories, a multi-frequency approach is necessary: 
- case B, the coating thickness is assumed to be known while the rib thickness is unknown and may take any of $N$ possible discrete values,

- case $\mathrm{C}$, the coating thickness is still known but the rib thickness is not and may take any value within a given range.

\subsection{Case B: $t_{r}$ can take $N$ possible values}

Here, we consider that the rib thickness $t_{r}$ can take $N$ discrete values $t_{r j}$ with $j \in\{1,2, \ldots, N\}$. Provided $N$ sets of linear characteristics (5) predetermined at $N$ frequencies $f_{i}$ with $i \in\{1,2, \ldots, N\}$, the measurement of $N I D_{i}$ at every $f_{i}$ enables computing the $N^{2}$ following estimated values:

$$
\hat{t}_{i j}=a_{i j}^{-1} \cdot N I D_{i} \quad i, j \in\{1,2, \ldots, N\}
$$

Let us call $j_{a c t}$ the index of the actual rib thickness of the inspected structure. When the index $j=j_{a c t}$, and in this case only, the air-gap $\hat{t}$ satisfies (9), which leads to its estimation.

$$
\hat{t}=a_{1 j_{a c t}}^{-1} \cdot N I D_{1}=a_{2 j_{a c t}}^{-1} \cdot N I D_{2}=\ldots=a_{N j_{a c t}}^{-1} \cdot N I D_{N}
$$

Let us consider the example $N=2$ with $t_{r 1}=1.5 \mathrm{~mm}$ and $t_{r 2}=25 \mathrm{~mm}$ as possible rib thicknesses. Consider 2 sets of curves (8) characterized at $f_{1}=680 \mathrm{~Hz}$ and $f_{2}=6600 \mathrm{~Hz}$ respectively. The normalized impedance distances $N I D_{1}$ and $N I D_{2}$ measured at $f_{1}$ and $f_{2}$ on an 
unknown configuration lead to the graphical identification (Figure 9) of the only $j$ value (in this case equal to 2) that satisfies (9). It follows:

$$
\hat{t}=a_{12}^{-1} \cdot N I D_{1}=a_{22}^{-1} \cdot N I D_{2}=300 \mu m, \text { with } \hat{t}_{r}=t_{r 2}=25 \mathrm{~mm} .
$$

More generally, considering $N$ possible rib thicknesses, we may build a vector $\mathbf{u}$ such that:

$$
\mathbf{u}=\frac{1}{N} \mathbf{A}^{\mathrm{T}} \mathbf{n}-\mathbf{t}
$$

in which $\mathbf{A}$ is the square matrix of the $a_{i j}^{-1}$ coefficients, $\mathbf{n}$ is a vector of the $N I D_{i}$ elements and $\mathbf{t}$ is the vector of the possible air-gap thicknesses $t_{i j}$, calculated at one in $N$ frequency $f_{i}$ and with $j \in\{1,2, \ldots, N\}$. The evaluation method then lies in the estimation of the actual index value $j_{a c t}$, according to:

$$
\hat{j}_{a c t}=\underset{1 \leq j \leq N}{\arg \min }\left(u_{j}\right)
$$

where $u_{j}$ is the $j^{\text {th }}$ element of the vector $\mathbf{u}$. Then, provided $\hat{j}_{a c t}$, the rib thickness $t_{r}$ and the airgap $t$ can be estimated as follows:

$$
\hat{t}_{r}=t_{r \hat{j}_{a c t}} \quad \text { and } \quad \hat{t}=\frac{1}{N} \sum_{i=1}^{N} t_{i \hat{j}_{a c t}}
$$

\subsection{Case $\mathrm{C}: t_{r}$ is unknown}


The air-gap estimation method proposed above can be generalized to case $\mathrm{C}$, for which $t_{r}$ is unknown. To do so, we build sets of linear characteristics $N I D(t)$ using simulated or measured data (Figure 10) obtained for discrete values of $t_{r}$. Then, using $k^{\text {th }}$-order polynomials, we extrapolate the values of the linear curves slopes (8) to that of any $t_{r}$ value comprised in the same range:

$$
a_{f}^{-1}\left(t_{r}\right)=c_{k} t_{r}^{k}+c_{k-1} t_{r}^{k-1}+\ldots+c_{2} t_{r}^{2}+c_{1} t_{r}+c_{0}
$$

In this study, we use polynomials of order $k=5$ so as to extrapolate the slopes $a_{f_{i}}^{-1}$ for layered structures featuring a coating thickness of $1.5 \mathrm{~mm}$ and rib thicknesses ranging from $1.5 \mathrm{~mm}$ to $3.5 \mathrm{~mm}$, and for a set of 5 frequencies ranging from $f_{1}=680 \mathrm{~Hz}$ to $f_{5}=2200 \mathrm{~Hz}$.

The estimated coefficients of these polynomials are gathered in Table 1. Provided these characteristics, it is possible to estimate the air-gap as follows: on every linear characteristic $N I D(t)$ parameterized by a given rib thickness $t_{r l}$, the $N I D_{i}$ value measured at $f_{i}$ corresponds to an air-gap $t_{i l}$ which is a possible solution of the problem:

$$
t_{i l}=a_{f_{i}}^{-1}\left(t_{r l}\right) . N I D_{i} \text { with } t_{r 1} \leq t_{r l} \leq t_{r L} \text { and } l \in\{1,2, \ldots, L\}
$$

where $t_{r 1}$ and $t_{r L}$ are the extreme values of the range of rib thicknesses $t_{r l}$ that is considered. Among the solutions provided by the equations (14), only those corresponding to the actual rib thickness, noted $t_{r l_{a c t}}$, will be true and also equal to each other. As a consequence, the actual rib thickness may be estimated as the one such that the equations (14) satisfy: 


$$
\hat{t}_{r l_{a c t}}=t_{r_{l_{a c t}}}
$$

where $\hat{l}_{a c t}$ is such that:

$$
t_{i \hat{l}_{\text {act }}}=\operatorname{argmin}\left\{\operatorname{std}\left(t_{i l}\right)\right\} \text { with } l \in\{1,2, \ldots, L\}
$$

where $\operatorname{std}($.$) denotes the standard deviation. Finally, the air-gap is estimated at every f_{i}$ frequency according to (17):

$$
\hat{t}=\frac{1}{N} \sum_{i=1}^{N} t_{\hat{i}_{a c t}}
$$

\section{Implementation of the evaluation methods}

In this section air-gap evaluations are carried out applying the methods proposed above either to simulated or experimental data.

\subsection{Implementation using simulated data}

Firstly, the proposed estimation algorithms are applied to simulated data provided by FE computations. The simulations are carried out for a fixed coating thickness $t_{c}=1.5 \mathrm{~mm}$, for the five following rib thicknesses $(1.5 \mathrm{~mm}, 2.0 \mathrm{~mm}, 2.5 \mathrm{~mm}, 3.0 \mathrm{~mm}, 3.5 \mathrm{~mm})$ and for the airgaps $(0,100 \mu \mathrm{m}, 200 \mu \mathrm{m}, \ldots, 500 \mu \mathrm{m})$. Moreover they are carried out for the five following EC frequencies $(680 \mathrm{~Hz}, 1060 \mathrm{~Hz}, 1440 \mathrm{~Hz}, 1820 \mathrm{~Hz}, 2200 \mathrm{~Hz})$. 
The sets of linear curves (4) constituting the direct behavioural model, which the estimation method relies on, are derived from the simulations. Furthermore, those done for the rib thickness $t_{r}=1.5 \mathrm{~mm}$ (which corresponds to the worst considered evaluation case) and for every considered air-gap, are chosen to build a set of test data. Moreover, in order to get close to practical implementation conditions which can be subject to measurement distortions, for example due to some sensor lift-off or tilt angle [16], a white Gaussian noise is added to the test data. Therefore, 1000 noise realizations are used for each considered air-gap value.

For the evaluation cases $\mathrm{A}, \mathrm{B}$ and $\mathrm{C}$ previously defined, that is for $t_{r}$ assumed to be known, for $t_{r}$ unknown among $N$ possible values and for $t_{r}$ unknown within a given range, the evaluation algorithms reported in sections $2.2,4.1$ and 4.2 are applied.

The estimation results obtained using test data featuring a $60 \mathrm{~dB}$ signal to noise ratio (SNR) are reported in Figure 11. Whether for cases A, B or C the estimates $\hat{t}$ vary linearly with a slope equal to 1 as a function of the actual air-gaps $t$ which tends to prove the estimation accuracy. However, the estimation accuracy can be quantified via the relative accuracy error RAE (18) and the relative precision error RPE (19):

$$
\begin{aligned}
& R A E \%=\text { mean }\left(\frac{|\hat{t}-t|}{t} \cdot 100\right) \\
& R P E \%=\text { mean }\left(\frac{\operatorname{std}(\hat{t})}{\operatorname{mean}(\hat{t})} \cdot 100\right)
\end{aligned}
$$

where mean(.) designates the mean value. Table 2 shows that for a $60 \mathrm{~dB}$ SNR, the estimation is accurate and precise since $0.04 \% \leq R A E \% \leq 0.66 \%$ and $0.04 \% \leq R P E \% \leq 0.93 \%$. 
The estimation results obtained using test data featuring an SNR of $33 \mathrm{~dB}$, are given in Figure 12. In the case A the estimation is accurate and also precise as RAE $\%<0.8 \%$ and RPE $\%<1 \%$, as shown in Table 2 . However, with regards to the cases B and $\mathrm{C}$, for which $t_{r}$ is unknown, the estimation is less accurate. Indeed, the curve representing $\hat{t}$ as a function of $t$ is still linear, but the slope is no longer equal to 1 but in the order of 0.9 . With regards to the estimation precision, it also decreases. Nevertheless the accuracy and the precision remain acceptable as $1.59 \% \leq R A E \% \leq 8,99 \%$ and $2.84 \% \leq R P E \% \leq 9.45 \%$.

Finally it is also to be noted (Table 2) that the larger $t_{r}$ the better the estimation accuracy and precision.

\subsection{Implementation on experimental data}

Secondly, the proposed estimation algorithms are applied to experimental data obtained using a layered structure featuring a coating thickness of $t_{c}=1.5 \mathrm{~mm}$ and a rib thickness of $t_{r}=1.5 \mathrm{~mm}$. The used air-gaps are again $\{0,100 \mu \mathrm{m}, 200 \mu \mathrm{m}, \ldots, 500 \mu \mathrm{m}\}$ and every considered structure configuration is inspected using the same set of five frequencies as used in section 5.1. Moreover, every measure is repeated 12 times, the sensor being re-positioned each time, which enables estimating the SNR of the experimental data using:

$$
S N R=10 \log \left(\frac{\left(\frac{1}{12} \sum_{n=1}^{12} N I D_{n}\right)^{2}}{\frac{1}{12} \sum_{n=1}^{12}\left(N I D_{n}-\frac{1}{12} \sum_{n=1}^{12} N I D_{n}\right)^{2}}\right)
$$

For the whole considered frequencies the experimental SNR appears to be $33 \mathrm{~dB}$. 
The estimation results obtained in the cases A, B and C are given in Figure 13 and in Table 2. They are in good agreement with those obtained using simulated data for the same structure configurations and for the same SNR.

\section{Conclusion}

In this paper we have proposed three methods for evaluating the thickness of the air-gap separating two conductive plates in the context of the EC NDE of the assembly of a coating on a wing rib or spar. The proposed methods rely on a multi-frequency behavioural model of the interactions between the coil sensor and the assembly to be evaluated.

Thanks to finite elements modeling simulations used to build a multi-frequency behavioural model, as well as test data, accurate and precise air-gap estimation results (in the worst considered cases the average value of the absolute relative errors and the relative standard deviation were $0.66 \%$ and $0.93 \%$ respectively) were obtained for data featuring a $60 \mathrm{~dB}$ signal to noise ratio, and for a 5 frequencies approach. Moreover, good estimation results were also obtained (in the worst considered cases the average value of the relative accuracy error and the relative precision error were $8.99 \%$ and $9.45 \%$ respectively) considering the same configurations but with test data featuring a $33 \mathrm{~dB}$ signal to noise ratio. Furthermore, the proposed methods were validated on experimental data.

The results analysis has shown that the larger the rib thickness, the better the estimation performance. When the rib thickness jumps from $1.5 \mathrm{~mm}$ to $3.5 \mathrm{~mm}$ the average value of the accuracy and precision errors thus decreases from $9 \%$ to $1.6 \%$ and from $9.5 \%$ to $2.9 \%$ respectively. Such a feature makes the proposed method promising for the considered aeronautical application because the rib and spar thicknesses are higher than $1.5 \mathrm{~mm}$. 
Future works will deal with the extension of the proposed analysis to thicker coatings as well as to assembled geometries closer to real configurations. The optimization of the choice of the frequencies and the use of the phase variations of the normalized impedance will be considered for the enhancement of the estimation. Also, further works will focus on the optimization of the sensor geometry so that it matches the geometry of real structures and enhances the electromagnetic coupling, in order to optimize the estimation performances.

\section{References}

1. Le Diraison Y., Joubert P.Y., Placko D., Characterization of subsurface defects in aeronautical riveted lap-joints using multi-frequency eddy current imaging, NDT\&E international 42 (2009), pp. 133-140.

2. Wrzuszczak M., Wrzuszczak J., Eddy current flaw detection with neural network applications, Measurement, Volume 38, Issue 2, September 2005, Pages 132-136.

3. Rosado L.S., Santos T.G., Piedade M., Ramos P.M., Vilaça P., Advanced technique for non-destructive testing of friction stir welding of metals, Measurement, In Press, Corrected Proof, Available online 13 February 2010.

4. Vernon S.N., The universal impedance diagram of the ferrite pot core eddy current transducer, IEEE trans magn 1999, 25(3):2639-45.

5. Huanga P., Zhanga G., Wub Z., Caia J., Zhou Z., Inspection of defects in conductive multi-layered structures by an eddy current scanning technique: simulation and experiments, NDT\&E international 39 (2006), pp. 578-584.

6. Pavo J., Gyimothy S., Adaptative inversion database for electromagnetic nondestructive evaluation, NDT \& E International 2007;40:192-202.

7. Cheng C.C., Dodds C.V., Deeds W.E., General analysis of probe coils near stratified conductors, ," Int. J. Nondestruct. Test., vol. 3, pp. 109-130, 1971. 
8. E. Uzal and J. H. Rose, "The impedance of eddy current probes over layered metals whose conductivity and permeability vary continuously, IEEE Trans. Magn., vol. 29, no. 2, pp. 1869-1873, Mar. 1993.

9. W. Yin, S. J. Dickinson, and A. J. Peyton, "Imaging the continuous conductivity profile within layered metal structures using inductance spectroscopy, IEEE Sensors J., pp. 161-166, 2005.

10. Renakos I.T., Theodoulidis T.P., Panas S.M., Tsiboukis T.D., Impedance inversion in eddy current testing of layered planar structures via neural networks, NDT\&E international, 30 (1997), pp. 69-74.

11. P. Huang, Z. Wu, "Inversion of thickness of multi-layered structures from eddy current testing measurements", SCIENCE (Journal of Zhenjiang University, China), vol. 5, no.1, pp. 86-91, Jan. 2004.

12. Le Bihan Y., Study on the Transformer Equivalent Circuit of Eddy Current Nondestructive Evaluation , NDT\&E International 36 (2003), pp. 297-302.

13. Libby H.L., Introduction to electromagnetic nondestructive test methods, New York: Wiley, 1971.

14. Cung T.L., Joubert P.-Y, Vourc'h E, Larzabal P., On the interactions of an eddy current sensor and a multilayered structure, Electron. Lett. Volume 46, Issue 23, p.1550-1551 (2010).

15. Yin W., Binns R., Davis C. and Peyton A. J. "Analysis of the lift-off effect of phase spectra for eddy current sensors", IEEE Trans. Instrumentation and Measurement Volume: 3, Page(s): $1779-1784$ (2005). 
16. Joubert P.Y., Le Bihan Y., Eddy Current Data Fusion for the Enhancement of Defect Detection in Complex Metallic Structures, International Journal of Applied Electromagnetics and Mechanics, vol. 19, n¹-4, 2004, pp. $647-651$. 


\section{Figures}

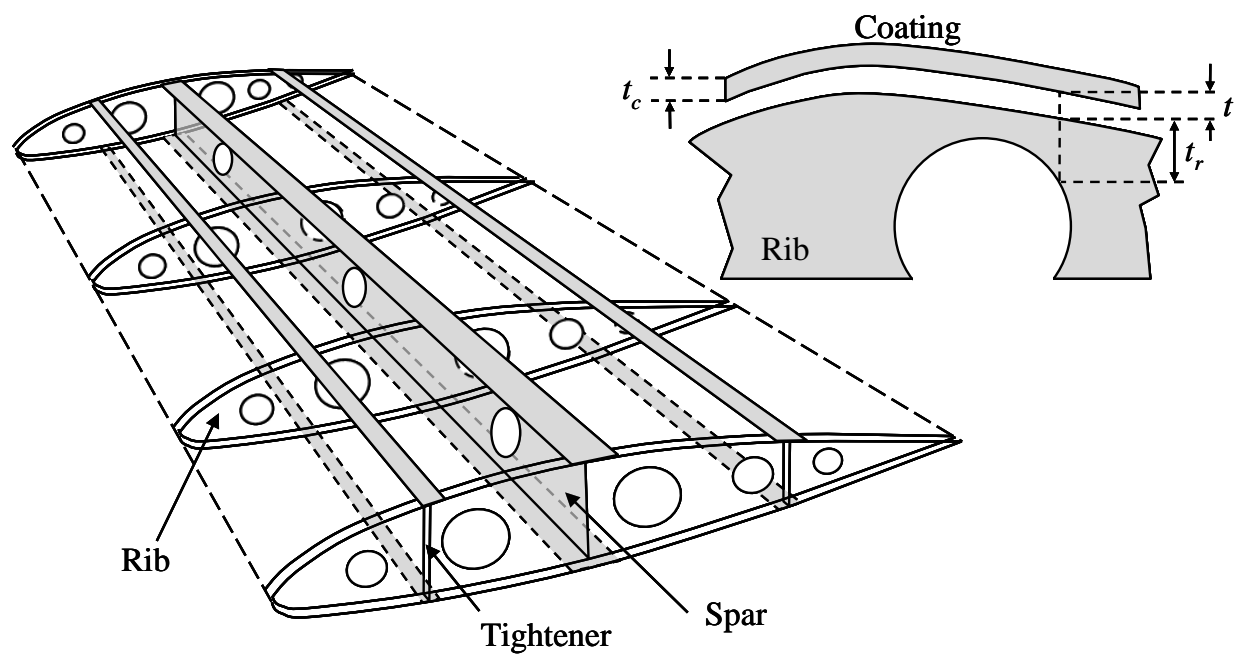

Figure 1. Structure of an aircraft wing and cut view of the assembly of a coating on a rib. 


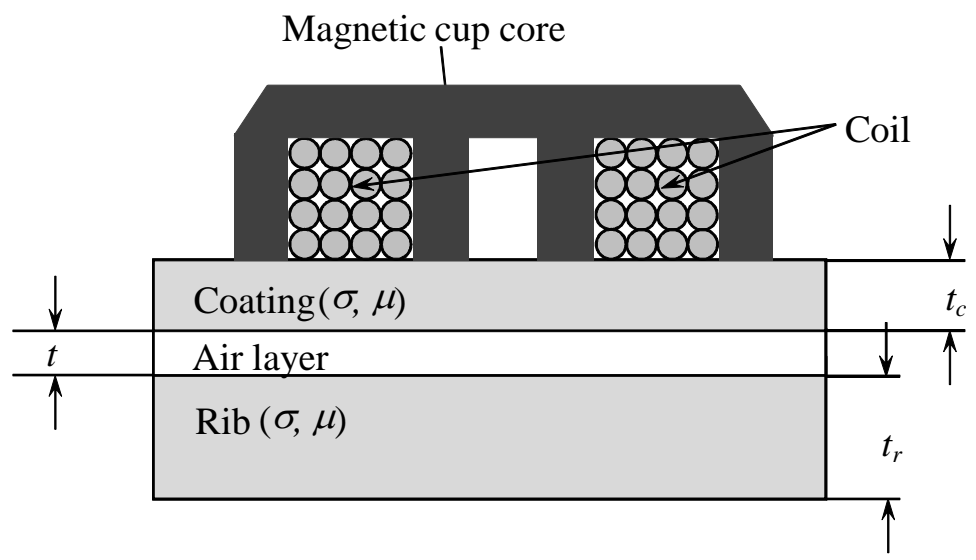

Figure 2. Eddy current cup core coil sensor placed on the inspected multilayered assembly. 


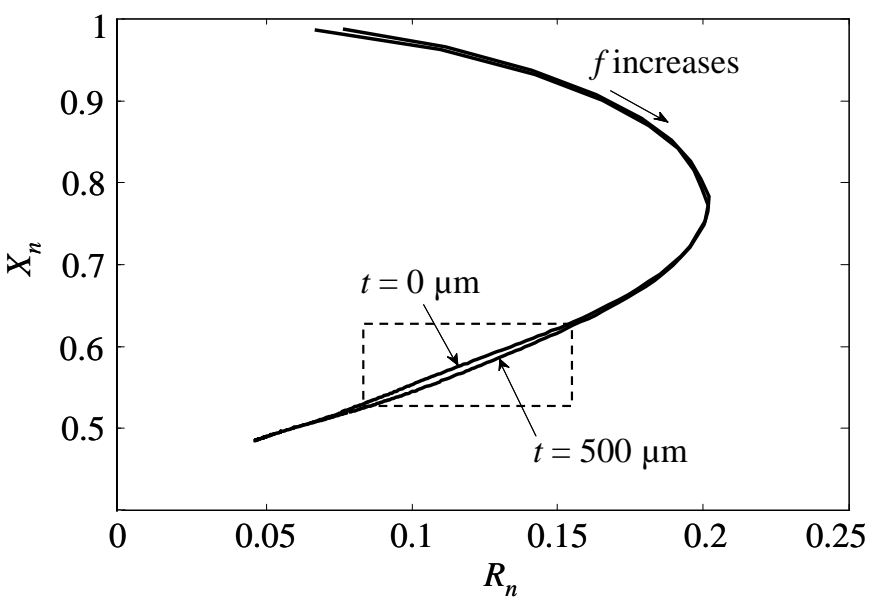

Figure 3. Universal impedance diagrams of the normalized impedance $Z_{n t}$ of the EC sensor placed on aluminium layers assemblies and measured in the $[80 \mathrm{~Hz}-30 \mathrm{kHz}]$ frequency range. The coating and the rib layers thicknesses are $t_{c}=1.5 \mathrm{~mm}$ and $t_{r}=25 \mathrm{~mm}$ respectively. The two measurements depicted correspond to the air-gaps $t=0$ and $t=500 \mu \mathrm{m}$. 


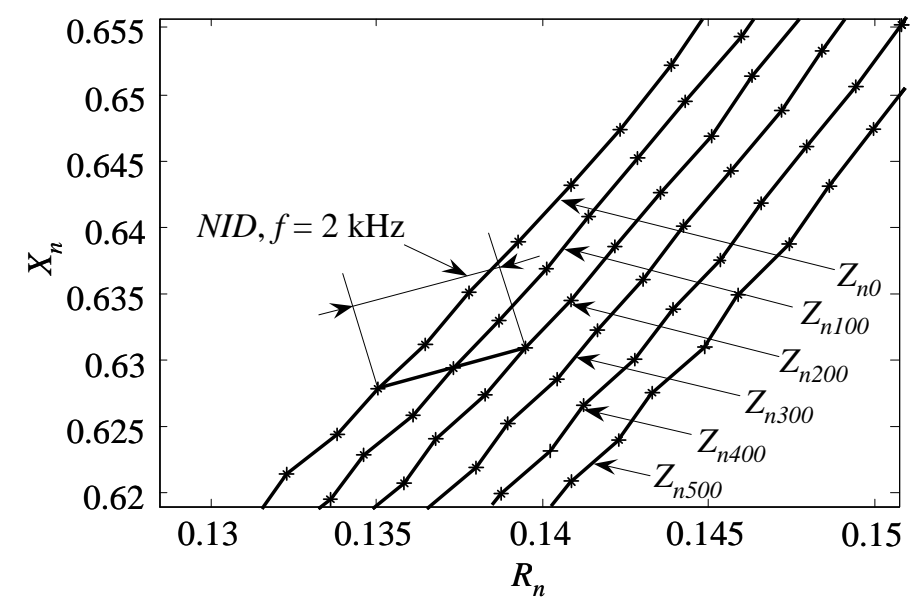

Figure 4. Normalized impedance $Z_{n t}(f)$ curves for $t$ ranging from 0 to $500 \mu \mathrm{m}$, $f \in[1550 \mathrm{~Hz} 2300 \mathrm{~Hz}], t_{c}=1.5 \mathrm{~mm}$ and $t_{r}=25 \mathrm{~mm}$. 


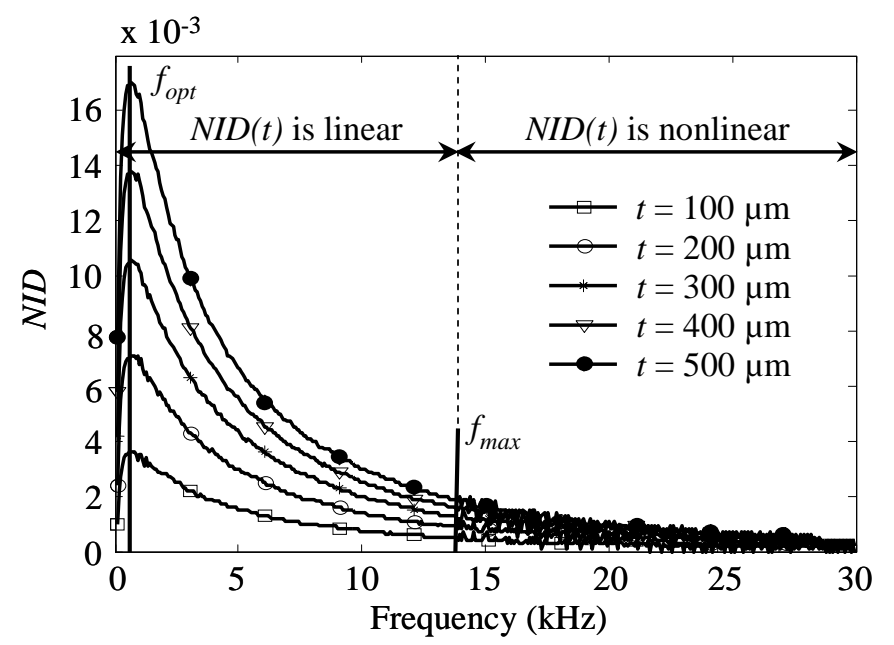

Figure 5. Variations of NID as a function of the excitation frequency $f$. The curves are parameterized by the air-gap $t \in\{100 \mu \mathrm{m}, 200 \mu \mathrm{m}, 300 \mu \mathrm{m}, 400 \mu \mathrm{m}, 500 \mu \mathrm{m}\}$. 


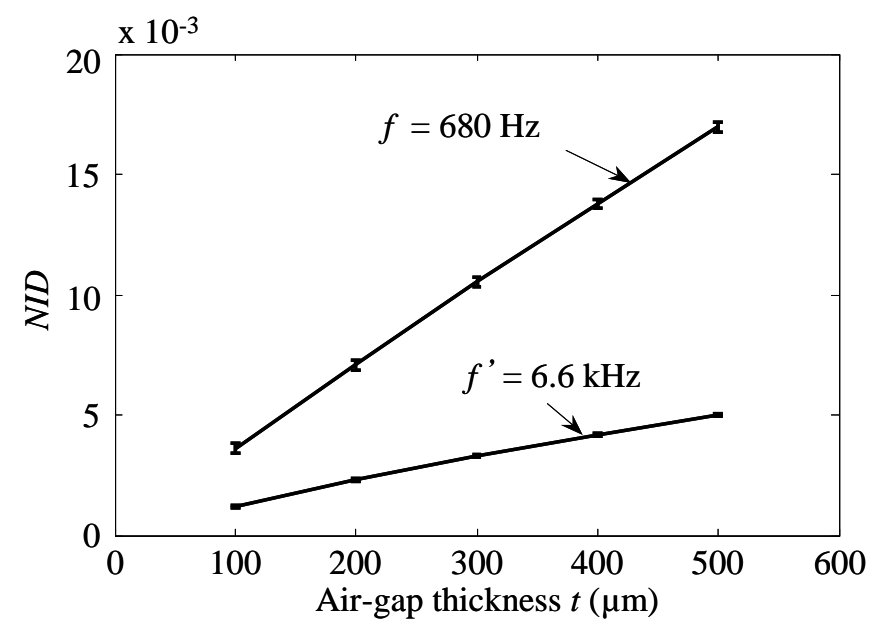

Figure 6. Linear relationship between NID and spacing $t$ implemented at two excited frequencies, where $t_{c}=t_{r}=1.5 \mathrm{~mm}$. The error bars correspond to a confidence interval of $95 \%$, estimated from a set of 12 measurements. 


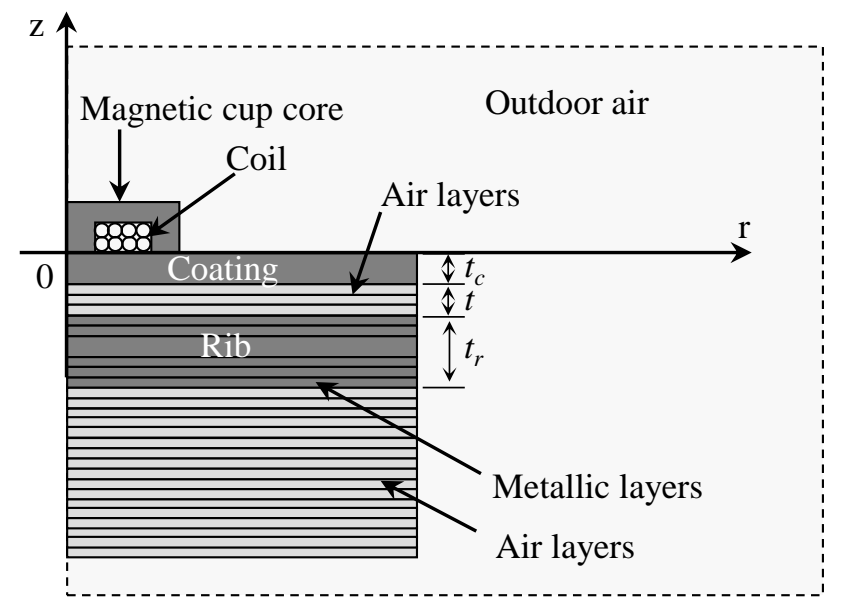

Figure 7. Two dimensional workspace of the finite element computations 


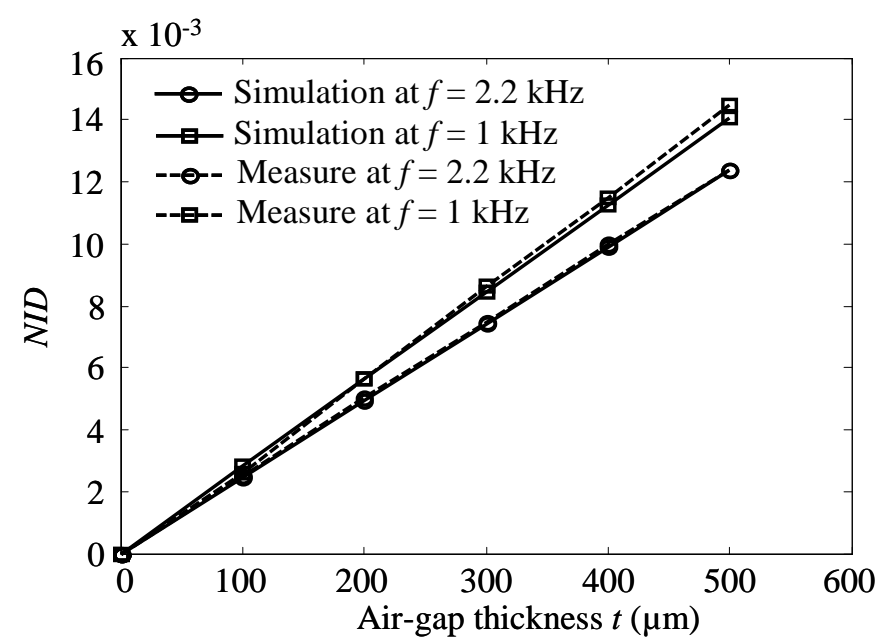

Figure 8. Linear variations of $N I D$ as a function of $t$ obtained experimentally and by FE simulations, for $t_{c}=t_{r}=1.5 \mathrm{~mm}$, for $f=1 \mathrm{kHz}$ and for $f=2.2 \mathrm{kHz}$. 


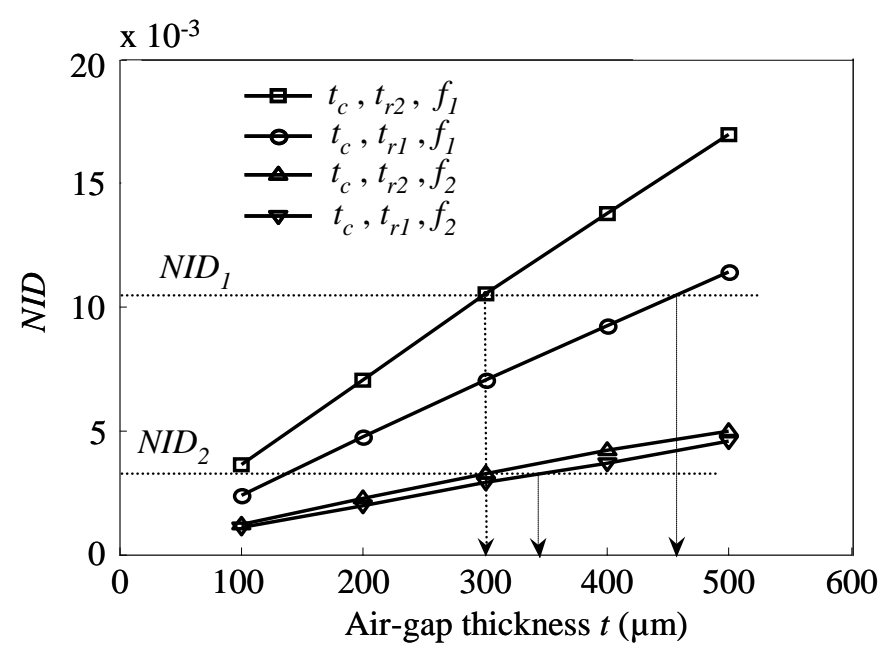

Figure 9. Set of two couples of linear characteristics $N I D(t)$, used for the bi-frequency $\left(f_{1}=680 \mathrm{~Hz}, f_{2}=6.6 \mathrm{kHz}\right)$ evaluation of $t, t_{c}$ being known and equal to $1.5 \mathrm{~mm}$ and $t_{r}$ being unknown but possibly equal to $t_{r 1}=1.5 \mathrm{~mm}$ or $t_{r 2}=25 \mathrm{~mm}$. 


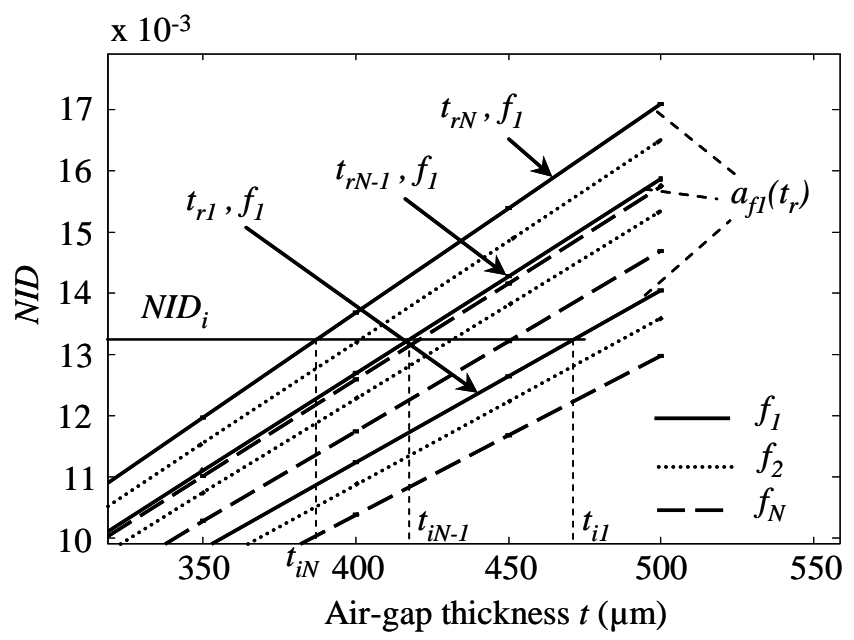

Figure 10. Set of linear curves $N I D(t)$. 

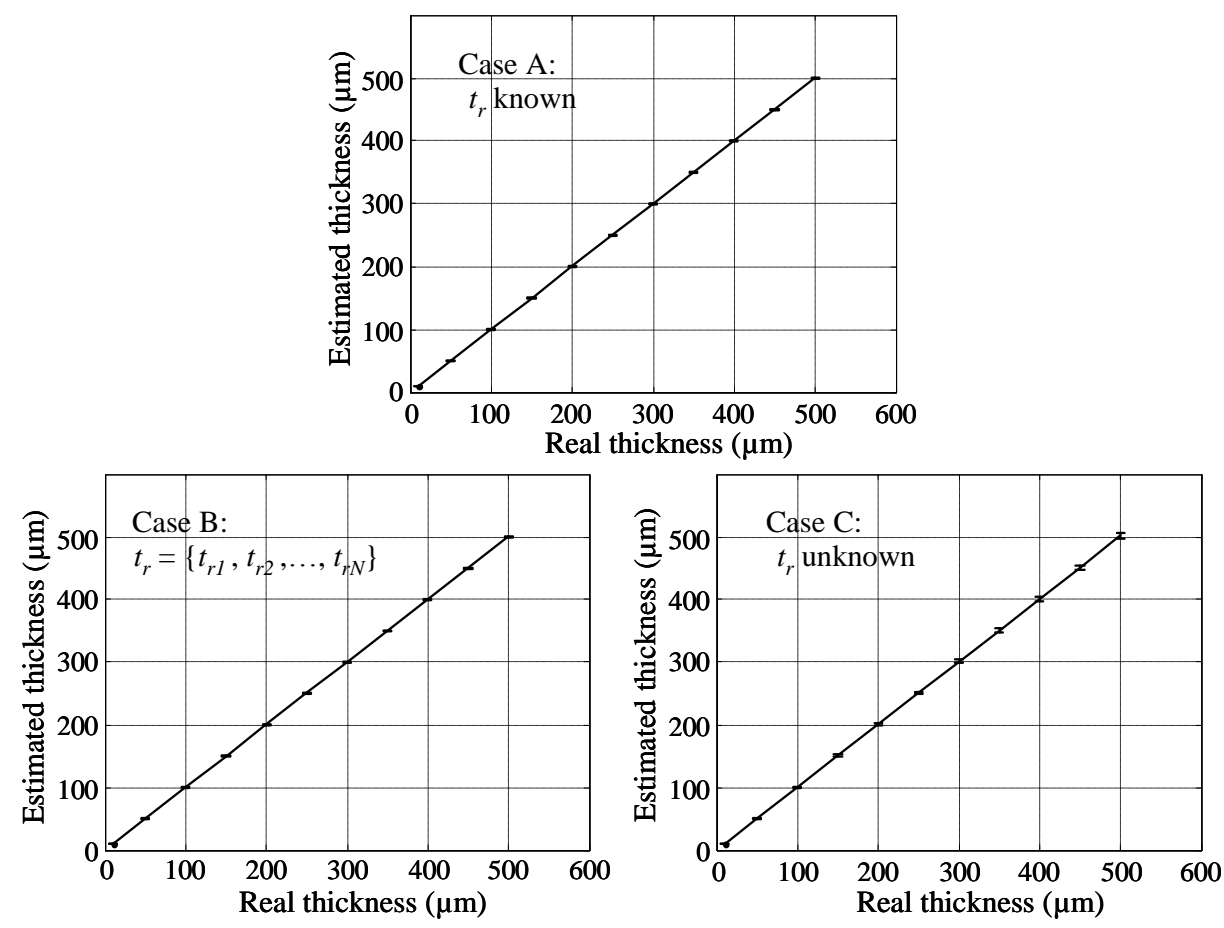

Figure 11. Results of the estimation of $t$ obtained with simulated data featuring a $60 \mathrm{~dB}$ SNR. Cases $t_{r}$ is known (case A), case $t_{r} \in(1,5 \mathrm{~mm}, 2 \mathrm{~mm}, 2.5 \mathrm{~mm}, 3 \mathrm{~mm}, 3.5 \mathrm{~mm})$ (case B), and case $t_{r}$ is unknown (case C). In every case, the actual rib thickness value is $1.5 \mathrm{~mm}$. 

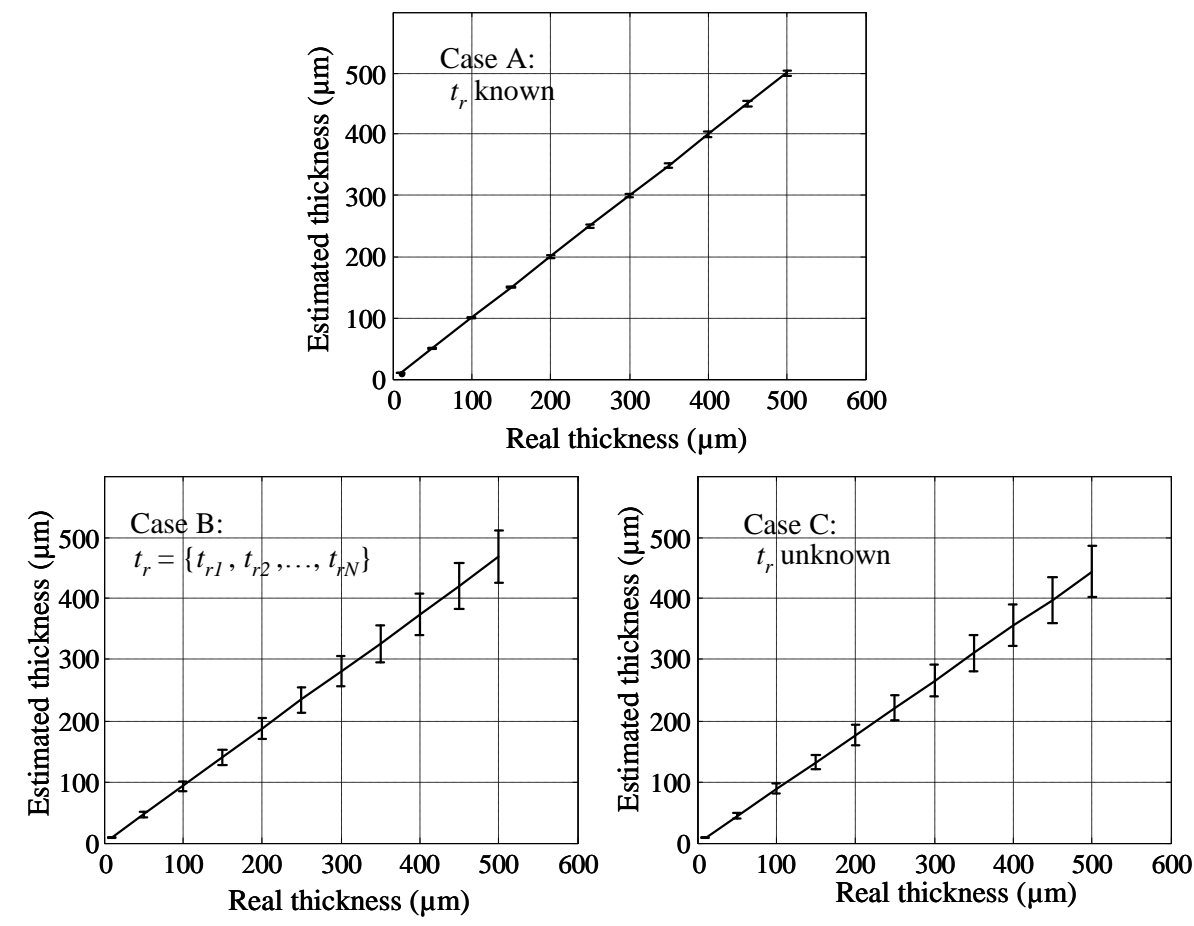

Figure 12. Results of the estimation of $t$ obtained with simulated data featuring a $33 \mathrm{~dB}$ SNR.

Cases $t_{r}$ is known (case A), case $t_{r} \in(1,5 \mathrm{~mm}, 2 \mathrm{~mm}, 2.5 \mathrm{~mm}, 3 \mathrm{~mm}, 3.5 \mathrm{~mm})$ (case B), and case $t_{r}$ is unknown (case C). In every case, the actual rib thickness value is $1.5 \mathrm{~mm}$. 

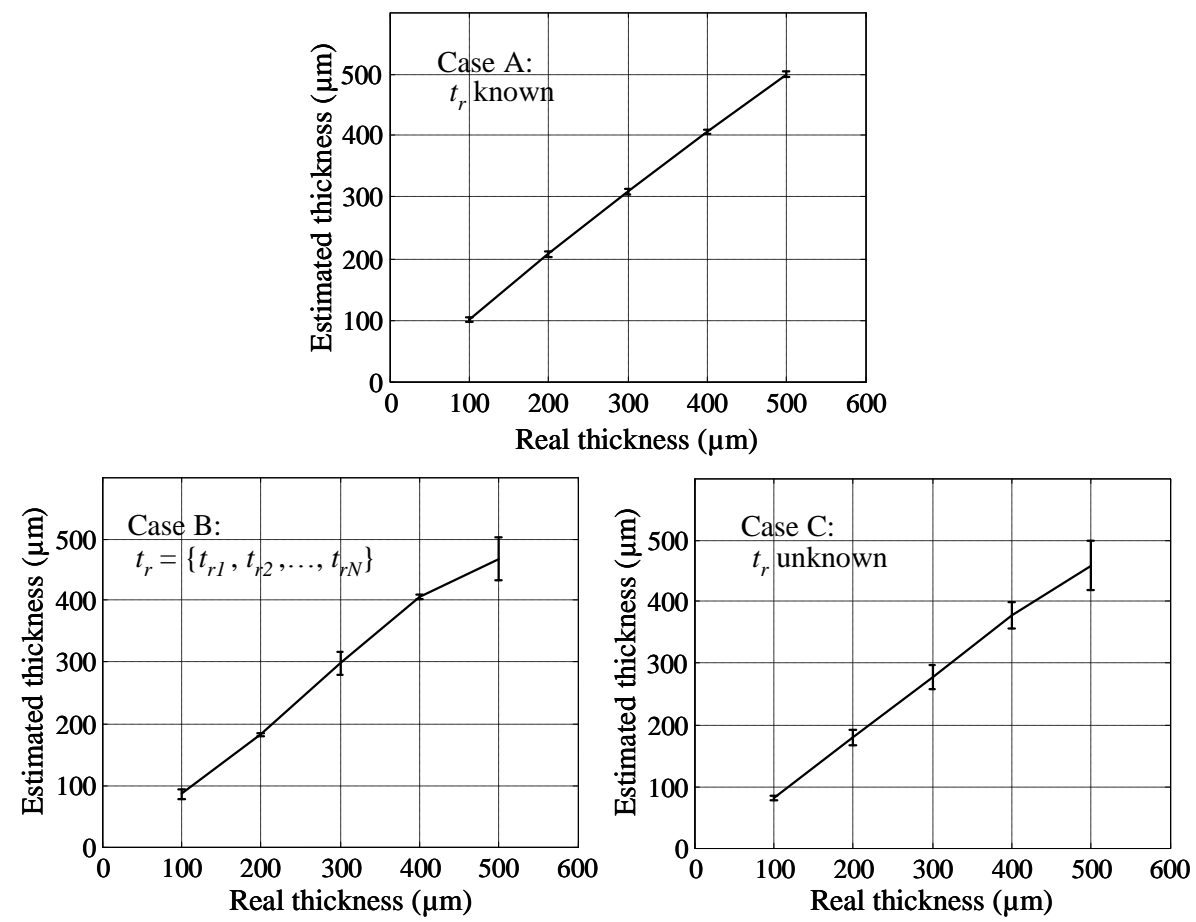

Figure 13. Results of the estimation of $t$ obtained with experimental data featuring a $33 \mathrm{~dB}$ SNR. Cases $t_{r}$ is known (case A), case $t_{r} \in(1,5 \mathrm{~mm}, 2 \mathrm{~mm}, 2.5 \mathrm{~mm}, 3 \mathrm{~mm}, 3.5 \mathrm{~mm})$ (case B), and case $t_{r}$ is unknown (case C). In every case, the actual rib thickness value is $1.5 \mathrm{~mm}$. 


\section{Tables}

\begin{tabular}{|c|c|c|c|c|c|c|}
\hline \multicolumn{7}{|c|}{$a_{f}^{-1}=c_{5} e_{n}^{5}+c_{4} e_{n}^{4}+c_{3} e_{n}^{3}+c_{2} e_{n}^{2}+c_{1} e_{n}+c_{0}$} \\
\hline \hline$f(\mathrm{~Hz})$ & $c_{5}\left(\times 10^{-4}\right)$ & $c_{4}\left(\times 10^{-3}\right)$ & $c_{3}\left(\times 10^{-2}\right)$ & $c_{2}\left(\times 10^{-2}\right)$ & $c_{1}$ & $c_{0}\left(\times 10^{-2}\right)$ \\
\hline 680 & 3.70 & 4.15 & 1.68 & 2.63 & 0 & 5.76 \\
\hline 1060 & 2.60 & 2.98 & 1.25 & 2.02 & 0 & 5.22 \\
\hline 1440 & 2.32 & 2.77 & 1.19 & 2.00 & 0 & 5.35 \\
\hline 1820 & 4.30 & 4.70 & 1.85 & 2.83 & 0 & 6.00 \\
\hline 2200 & 2.76 & 3.32 & 1.44 & 2.38 & 0 & 6.01 \\
\hline
\end{tabular}

Table 1. Coefficients of the five $5^{\text {th }}$-order polynomials extrapolated at the 5 considered frequencies. 


\begin{tabular}{|c|c|c|c|}
\hline \multicolumn{4}{|c|}{ Actual plates thicknesses $t_{c}=1.5 \mathrm{~mm} ; t_{r}=1.5 \mathrm{~mm}$} \\
\hline Data & $\begin{array}{c}\text { Case } \mathrm{A} \\
(R P E \%-R A E \%)\end{array}$ & $\begin{array}{c}\text { Case B } \\
(R P E \%-R A E \%)\end{array}$ & $\begin{array}{c}\text { Case } \mathrm{C} \\
(R P E \%-R A E \%)\end{array}$ \\
\hline Simulated $(S N R=60 \mathrm{~dB})$ & $0.04-0.04$ & $0.04-0.04$ & $0.93-0.66$ \\
\hline Simulated $(S N R=33 \mathrm{~dB})$ & $0.99-0.79$ & $9.04-6.94$ & $9.45-8.99$ \\
\hline Experimental $(S N R \approx 33 \mathrm{~dB})$ & $1.79-2.49$ & $7.45-7.79$ & $6.60-9.42$ \\
\hline \multicolumn{4}{|c|}{ Actual plates thicknesses $t_{c}=1.5 \mathrm{~mm} ; t_{r}=3.5 \mathrm{~mm}$} \\
\hline Data & $\begin{array}{c}\text { Case } \mathrm{A} \\
(R P E \%-R A E \%)\end{array}$ & $\begin{array}{c}\text { Case B } \\
(R P E \%-R A E \%)\end{array}$ & $\begin{array}{c}\text { Case } \mathrm{C} \\
(R P E \%-R A E \%)\end{array}$ \\
\hline Simulated $(S N R=60 \mathrm{~dB})$ & $0.04-0.04$ & $0.04-0.04$ & $0.06-0.05$ \\
\hline Simulated $(S N R=33 \mathrm{~dB})$ & $0.93-0.76$ & $5.20-2.85$ & $2.84-1.59$ \\
\hline
\end{tabular}

Table 2. The RPE and RAE of the estimation results 


\section{Figure captions}

Figure 1. Structure of an aircraft wing and cut view of the assembly of a coating on a rib.

Figure 2. Eddy current cup core coil sensor placed on the inspected multilayered assembly.

Figure 3. Universal impedance diagrams of the normalized impedance $Z_{n t}$ of the EC sensor placed on aluminium layers assemblies and measured in the $[80 \mathrm{~Hz}-30 \mathrm{kHz}]$ frequency range. The coating and the rib layers thicknesses are $t_{c}=1.5 \mathrm{~mm}$ and $t_{r}=25 \mathrm{~mm}$ respectively. The two measurements depicted correspond to the air-gaps $t=0$ and $t=500 \mu \mathrm{m}$.

Figure 4. Normalized impedance $Z_{n t}(f)$ curves for $t$ ranging from 0 to $500 \mu \mathrm{m}$, $f \in[1550 \mathrm{~Hz} 2300 \mathrm{~Hz}], t_{c}=1.5 \mathrm{~mm}$ and $t_{r}=25 \mathrm{~mm}$.

Figure 5. Variations of $N I D$ as a function of the excitation frequency $f$. The curves areparameterized by the air-gap $t \in\{100 \mu \mathrm{m}, 200 \mu \mathrm{m}, 300 \mu \mathrm{m}, 400 \mu \mathrm{m}, 500 \mu \mathrm{m}\}$.

Figure 6. Linear relationship between NID and spacing $t$ implemented at two excited frequencies, where $t_{c}=t_{r}=1.5 \mathrm{~mm}$. The error bars correspond to a confidence interval of $95 \%$, estimated from a set of 12 measurements.

Figure 7. Two dimensional workspace of the finite element computations

Figure 8. Linear variations of $N I D$ as a function of $t$ obtained experimentally and by FE simulations, for $t_{c}=t_{r}=1.5 \mathrm{~mm}$, for $f=1 \mathrm{kHz}$ and for $f=2.2 \mathrm{kHz}$. 
Figure 9. Set of two couples of linear characteristics $N I D(t)$, used for the bi-frequency $\left(f_{1}=680 \mathrm{~Hz}, f_{2}=6.6 \mathrm{kHz}\right)$ evaluation of $t, t_{c}$ being known and equal to $1.5 \mathrm{~mm}$ and $t_{r}$ being unknown but possibly equal to $t_{r 1}=1.5 \mathrm{~mm}$ or $t_{r 2}=25 \mathrm{~mm}$.

Figure 10. Set of linear curves $N I D(t)$.

Figure 11. Results of the estimation of $t$ obtained with simulated data featuring a $60 \mathrm{~dB}$ SNR. Cases $t_{r}$ is known (case A), case $t_{r} \in(1,5 \mathrm{~mm}, 2 \mathrm{~mm}, 2.5 \mathrm{~mm}, 3 \mathrm{~mm}, 3.5 \mathrm{~mm})$ (case B), and case $t_{r}$ is unknown (case C). In every case, the actual rib thickness value is $1.5 \mathrm{~mm}$.

Figure 12. Results of the estimation of $t$ obtained with simulated data featuring a $33 \mathrm{~dB}$ SNR. Cases $t_{r}$ is known (case A), case $t_{r} \in(1,5 \mathrm{~mm}, 2 \mathrm{~mm}, 2.5 \mathrm{~mm}, 3 \mathrm{~mm}, 3.5 \mathrm{~mm})$ (case B), and case $t_{r}$ is unknown (case C). In every case, the actual rib thickness value is $1.5 \mathrm{~mm}$.

Figure 13. Results of the estimation of $t$ obtained with experimental data featuring a $33 \mathrm{~dB}$ SNR. Cases $t_{r}$ is known (case A), case $t_{r} \in(1,5 \mathrm{~mm}, 2 \mathrm{~mm}, 2.5 \mathrm{~mm}, 3 \mathrm{~mm}, 3.5 \mathrm{~mm})$ (case B), and case $t_{r}$ is unknown (case C). In every case, the actual rib thickness value is $1.5 \mathrm{~mm}$. 